\title{
A Corpus-Based Study of Adverbial Connectors in Native and Non-native Students' Writing*
}

\author{
WANG Yan-jun, LI Rui \\ College of Chinese Language and Culture, Jinan University, Guangzhou City, China
}

\begin{abstract}
In writing and speaking, adverbial connectors always play a part to connect different parts together at both semantic and syntactic aspects. For it is so important that a lot of scholars have made and are making a series of studies to their functions. On the basis of corpus-based study, this paper firstly makes a comparison to the usage of adverbial connectors in their writings of the native students and non-native students and then exposes a distinctive gap between these two types of writings in usage of adverbial connectors. In order to help Chinese second-language learners acquire English as well as English native speakers do, this study deeply digs this gap and in further provides several pedagogical suggestions for English teachers and second-language learners.
\end{abstract}

Keywords: corpus, adverbial connector, students’ writing, cohesion

\section{Brief Introduction to the Research}

Adverbial connectors are generally defined as words or phrases that can help connect each sentence together. M. A. K. Halliday holds the belief that adverbial connectors serve the textural and interpersonal functions of language in his S-F Grammar (Halliday, 1994, pp. 323-327). That means, in writing, adverbial connectors can not only help writers link sentences together, but also in reading aid readers to comprehend the text as little difficult as possible. From this perspective, in writing and comprehending, adverbial connectors, the signals of the complex logical relations of written discourse, can both make an influence to writers and readers.

In the past several decades, plenty of researches have attached importance to this linguistic phenomenon that the usage of connectors is problematic for language learners, especially foreign language learner. In general, the results of these researches can be concluded into four kinds. First of all, adverbial connectors are not always needed in writing. Secondly, the use of connectors is sensitive to register and discourse type. Thirdly, many connectors are multifunctional, that is, they perform different functions in different contexts. The last one is that the usage of connectors tends to vary from one language to another. Hence, with the view of doing a further research, the article tries to use two corpora CLEC and FLOB, to investigate how Chinese second-language learners and native English speakers employ adverbial connectors in their writings.

\footnotetext{
* Acknowledgements: This paper has been sponsored by Jinan University; and this study is supported by the Project of National Social Science Fund in 2012: Research on the Potential Construction in Northern Dialects Since Yuan Dynasty. Notes: Data of all these tables are obtained from the concordance of two corpora by means of Antconc.

WANG Yanjun, Doctor in Linguistics, Professor, Doctorial Supervisor, College of Chinese Language and Culture, Jinan University.

LI Rui (Corresponding Author), Ph.D. Student, College of Chinese Language and Culture, Jinan University.
} 
Through this investigation, this study aims to give some tentative suggestions to second language learners about how to employ this type of linguistic resources in a more effective way, what the difference is to the usage of these adverbial connectors between Chinese English learners and native English learners and what the general tendency in their usage is, and even what the overused and underused items by Chinese second-language learners are, and moreover this paper tries to throw some light on the teaching practice at this respect.

\section{Definition of Adverbial Connector}

Briefly speaking, adverbial connectors refer to words or phrases that connect the meaning of one sentence or clause with the idea of another. Halliday names them as conjunctions. "It is a kind of semantic relation which functions as a specification of the way in which what is to follow is systematically connected to what has gone before” (Halliday \& Hasan, 1976, p. 227). This definition means that conjunctions or adverbial connectors help to connect two parts together while they express a type of semantic relations between different elements in clauses. In fact, besides this definition, there are still others. In this article, in the consideration of acceptability of most readers, "adverbial connector" is chosen to use.

In the consideration of expressive forms and other elements, adverbial connectors can be classified into five major types including single adverbs (e.g., however, therefore, etc.), adverb phrases (e.g., even so, etc.), prepositional phrases (e.g., in particular, for instance, etc.), finite clauses (e.g., that is, that is to say, etc.) and non-finite clauses (e.g., to conclude, to sum up, etc.). Besides this main categorization, there are still other types of classification to them from different perspectives by different schools, such as Biber's (Biber, et al 2000), Halliday’s (Halliday, et al 1976), Quirk’s (Quirk, 1972), Kopple’s (Kopple, 1980) classification and so on. In this article, in order for convenient discussion, Biber's classification is chosen as a basic one supplemented by others' in each detail. Biber named adverbial connectors as linking adverbials, which serve to state the writers' perception of the relationship between two units of discourse rather than adding additional information to a clause. They are important devices for making text cohesive, alongside coordinators and subordinators. Biber proposed a list of six types of logical relations which can be realized by adverbial connectors, including: enumeration and addition (e.g., firstly, at the beginning, in addition and so on), summation (e.g., in sum, to conclude and so forth), apposition (e.g., in other words, for example, that is and so on), result/inference (so, therefore, thus, and so forth), contrast/concession (e.g., alternatively, on the other hand, though and so on), transition (e.g., now, by the way and so forth) (cf. Biber, 2000, p. 103). By corroboration, the basic classification is accomplished in the following Table 1 :

Table 1

Classification of Adverbial Connectors

\begin{tabular}{|l|l|l|}
\hline $\begin{array}{l}\text { Semantic } \\
\text { classification }\end{array}$ & Adverbial connectors & $\begin{array}{l}\text { Functional } \\
\text { classification }\end{array}$ \\
\hline $\begin{array}{l}\text { Enumeration \& } \\
\text { addition }\end{array}$ & $\begin{array}{l}\text { Firstly, secondly, thirdly, ... } \\
\text { First of all, to begin with, next, then, finally, lastly, last but not least, equally, and, in the } \\
\text { same way, also, moreover, too, what is more, likewise, similarly, further, furthermore, in } \\
\text { addition, besides, in particular, above all. }\end{array}$ \\
\hline Summation & $\begin{array}{l}\text { Overall, all in all, in sum, in summary, to summarize, generally speaking, in brief, in } \\
\text { conclusion, to sum up, in general, on the whole, in short, briefly, in a word, so far. }\end{array}$ \\
\hline Apposition & $\begin{array}{l}\text { Namely, that is, that is to say, in other words, for instance, or rather, for example, for } \\
\text { instance, specifically. }\end{array}$ \\
\hline
\end{tabular}


(table 1 continued)

\begin{tabular}{|c|c|c|}
\hline \begin{tabular}{|l|} 
Semantic \\
classification
\end{tabular} & Adverbial connectors & \begin{tabular}{|l|}
$\begin{array}{l}\text { Functional } \\
\text { classification }\end{array}$ \\
\end{tabular} \\
\hline $\begin{array}{l}\text { Contrast \& } \\
\text { concession }\end{array}$ & $\begin{array}{l}\text { Rather, on the other hand, conversely, instead, on the contrary, in contrast, by contrary, by } \\
\text { comparison, anyway, nonetheless, however, nevertheless, at any rate, after all, needless to } \\
\text { say, notwithstanding, but, at the same time, still, though, yet, in any case, in any event, } \\
\text { admittedly, otherwise. }\end{array}$ & \\
\hline $\begin{array}{l}\text { Result \& } \\
\text { inference }\end{array}$ & $\begin{array}{l}\text { Accordingly, hence, as a result, in that case, finally, in this way, consequently, so, therefore, } \\
\text { thus, as a consequence, as the result, somehow, or else, otherwise, then, in other words, } \\
\text { subsequently, by the same token. }\end{array}$ & \multirow[t]{2}{*}{$\begin{array}{l}\text { Textual adverbial } \\
\text { connectors }\end{array}$} \\
\hline Transition & Incidentally, meanwhile, in the meantime, well, now. & \\
\hline Corroboration & Actually, in fact, as a matter of fact, in effect, indeed, apparently, in actual fact, of course. & $\begin{array}{l}\text { Interpersonal } \\
\text { adverbial } \\
\text { connectors } \\
\end{array}$ \\
\hline
\end{tabular}

From this table, we can find that these six types of adverbial connectors are not only different in the logical aspect, but all in semantic and functional aspects. All these factors can make effect on their usages in writing. In the following analysis, such difficulty in writing, causing the gap between two types of students, will be exploded.

\section{The Analysis of and the Discussion to the Relevant Data}

\section{Overall Frequencies of Adverbial Connectors}

After a careful checkup of the concordance of each adverbial connector, the total frequency of adverbial connectors in these two corpora is listed respectively in the Table 2.

Table 2

Total Frequency of Adverbial Connectors

\begin{tabular}{llll}
\hline Corpora & Total word & No. of texts & No. of connectors \\
\hline CLEC & $1,000,000$ & 5 & 33,829 \\
FLOB & $1,000,000$ & 15 & 22,936 \\
\hline
\end{tabular}

Here, Table 2 tells that adverbial connectors occur with a higher frequency in CLEC than in FLOB, among which the Chinese English learners use 33,738 adverbial connectors in a 1,000,000 word corpus while the native speakers 22,936 adverbial connectors in a corresponding 1,000,000 word corpus. It is obvious that Chinese second-language learners employ more connectors in writing while the native learners do less. It implies that Chinese second-language learners, when expressing connective meanings, intend to use these words rather than other words of expressing potential meaning. When connecting different elements, Chinese learners attempt to choose the similar adverbial connectors which are equivalent with Chinese translational versions in a sense. Due to such cognition of adverbial connectors, more and more connectors are used by Chinese writers in writing and speaking.

In the following, the research focus transfers to frequency distribution of different semantic categories of adverbial connectors. We make a categorization to all adverbial connectors into different groups, on the basis of the logical relation they represent. Finally, two frequency lists are illustrated with regard to the occurrence of categories of connectors and even their rank order in the following Table 3: 
Table 3

Quantity and Rank Order of Adverbial Connectors

\begin{tabular}{lll}
\hline Semantic relation & CLEC & FLOB \\
\hline Enumeration and addition & 7,898 & 5,133 \\
Summation & 329 & 291 \\
Apposition & 1,356 & 708 \\
Result and inference & 9,718 & 4,801 \\
Contrast and concession & 1,0179 & 8,441 \\
Transition & 3,166 & 2,555 \\
Corroboration & 1,092 & 1,007 \\
Total & 33,829 & 22,936 \\
\hline
\end{tabular}

In the Table 3, it shows: first, the distribution of different semantic categories in these two corpora is roughly the same. In general, enumeration and addition, result and inference, contrast and concession, and transition are more frequently used in writing, while summation, apposition and corroboration are used less frequently in writing. Then, though there is general overuse of all semantic categories of adverbial connectors in Chinese second-language learners' writings, the using of the categories of corroboration and summation is the same in two different types of learners. Among these two groups of data, the most obvious contrast occurs in enumeration and addition, result and inference, and contrast and concession. That means, in writing, Chinese students more intend to use such types of words or phrases to express the conclusion or conversion at semantic aspect.

\section{Adverbial Connectors Most Frequently Used in the Two Groups}

By ranking all these connectors according to their frequency of occurrence in a descending order, two respective frequency lists of the top 10 connectors for the two corpora are obtained, illustrated in the following Table 4:

Table 4

Frequency Lists of the Top Ten Connectors

\begin{tabular}{llllll}
\hline CLEC & Number & $\%$ & FLOB & Number & $\%$ \\
\hline so & 6,435 & 19.02 & then & 1,497 & 6.53 \\
but & 6,128 & 18.11 & also & 1,298 & 5.66 \\
also & 2,579 & 7.62 & still & 884 & 3.84 \\
then & 1,839 & 5.34 & too & 833 & 3.63 \\
however & 1,690 & 5.00 & however & 661 & 2.88 \\
though & 1,606 & 4.75 & though & 538 & 2.35 \\
still & 1,016 & 3.00 & rather & 461 & 2.01 \\
for example & 864 & 2.55 & next & 453 & 1.98 \\
in fact & 502 & 1.43 & yet & 421 & 1.84 \\
that is & 379 & 1.12 & of course & 318 & 1.39 \\
total & 23,038 & 68.10 & total & 7,364 & 32.10 \\
\hline
\end{tabular}

First of all, the table shows that half of these listed ten adverbial connectors are identical in the two corpora, although their rank order differs somewhat. Most evident differences occur in also and then. The word "also" is ranked number 3, occurring 2,597 times in CLEC while it is ranked number 2 occurring 1,298 times in FLOB. The word "then" is ranked number 4, occurring 1,839 times in CLEC, while it is ranked number 1 
occurring 1,497 times in FLOB. Of course, there are still some adverbial connectors which two corpora do not share each other among these top ten connectors. By observing the data, the words "so" and "but" are respectively ranked number 1 and number 2 in CLEC, but do exist among the top ten in the FLOB.

Secondly, in CLEC, the top ten connectors represented 68.10 percent of the total number of connector tokens, while in FLOB, they represent just 32.10 percent. On one side, it is obvious that the Chinese second-language learners intend to use more adverbial connectors to express their connecting meanings in writing, but the native learners are used to use connectors less frequently in writing. Even if adverbial connectors are used in writing, no one is specifically used more times than others. On the other side, Chinese English learners intend to use such words which are very familiar with them and to those unfamiliar words, they consciously or unconsciously neglect in writing. This phenomenon reflects the language acquisition process of Chinese second-language learners.

\section{Discussion to the Former Tables}

In the Table 2: two corpora of same size, Chinese learners employ more adverbial connectors than native speakers. One possible explanation could be that Chinese teachers place too much emphasis on connectors in constructing sentences and paragraphs. Actually, from the very beginning, Chinese second-language learners are required to pay attention to applying various adverbial connectors to transition in writing. They are cultivated to employ adverbial connectors as tools to mark various relationships between sentences and paragraphs. But, the emphasis of such instruction tends to cause negative consequences, that is, the mechanical use. The learners misunderstand that the more connectives they used and the fewer mistakes they made, the more coherent the writing would be and the higher scored they would achieve in the test.

Another possibility might be due to certain insecurity among non-native speakers regarding the effectiveness of their own writing. Anxious to make an impression and owing to their limited linguistic proficiency, they might feel a greater need than native speakers to stress the relevance of what they have to say.

The phenomenon appearing in the Table 3 can be proved in the actual writing. The using of enumeration and addition, result and inference, and contrast and concession is more frequent than the other ones. Learners tend to express these kinds of viewpoints by these words rather than the others. In a sense, it is caused by traditional English teaching method in China. No matter when in writing or reading, such kinds of words or phrases are overemphasized by both teachers and students.

In the Table 4, the fact of using same adverbial connectors means that both the Chinese English learners and the native learners rely on roughly these same connectors to accomplish their writing. Due to these data collected from the senior English learners, it could be assumed that learners tend to gradually strengthen their ability of applying this group of linguistic resources along with their progress in language proficiency. Advanced learners are quite aware of and capable of applying adverbial connectors to assist their writings and express various sentential relations.

Then, it is worth noting that the tendency to exploit a limited set of items is more frequent in Chinese English learners than in native learners: the top ten adverbial connectors represent $68.10 \%$ in CLEC, but just $32.10 \%$ in FLOB. The possible explanation is that Chinese English learners own relatively small word power and they are accustomed to using their most familiar and small number of adverbial connectors. They probably avoid applying certain connectors in which they had less confidence due to their lack of understanding of the abstract meaning of some items or due to their inexperience in using them. In contrast to Chinese English 
learners, the native learners own more word power and they are more familiar of these abstract words. Hence, they can use more different adverbial connectors.

\section{Conclusion}

\section{Important Findings of the Research}

Through qualitative and quantitative analysis of a large quantity of authentic language data, which is best facilitated by the powerful corpus processing software package Antconc, the research results have provided answers to the research questions and also revealed some problems in the writing by comparing two corpora: CLEC, FLOB.

Fist of all, Chinese English learners have displayed a general tendency to use more connectors in their writing. Due to instruction and linguistic proficiency, Chinese English learners overuse a lot of adverbial connectors which reveal certain insecurity among non-native learners regarding the effectiveness of their own writing.

Secondly, the result shows that the Chinese learner intends to overuse some words like: so, but, still and so on which might be decided by their familiarity to these words.

Thirdly, the research implies that there might be influence of Chinese mode of thinking on the Chinese learners' use of adverbial connectors. For instance, the Chinese English learners adopt inductive method of reasoning in their English writing, which might be effected by their Chinese mode of thinking and Chinese writing patterns; as a result, they tend to overuse such items so, in this way, therefore and so on which might be equivalent with the Chinese words suo yi, yi ci and zhe yang, etc.

\section{Pedagogical Implications of the Present Research}

Chinese second-language learners possess considerable awareness of applying adverbial connectors to assist their organization of writings. However, due to their mechanical use, excessive use, and misuse of adverbial connectors, they can not still master these words. Hence, they should improve more their second language proficiency. The followings are some advices obtained from this research.

To begin with, the study shows that teachers should avoid overstressing the value of adverbial connectors while raising learners' awareness of applying adverbial connectors. Teachers need to ensure that learners do not go to extreme, using connectors excessively and willfully without a full consideration of the appropriateness and accuracy. Learners should put more of their attention on the messages they want to convey rather than the form when they write, and also they need to be encouraged to seek for flexibility in the way of constructing texts.

Secondly, the study shows that Chinese second-language learners should be aware that learning the native English writing conventions is inextricable from learning to write in English. Learners should acquire a better understanding of the English writing conventions and patterns. They are expected to present their arguments in the English way.

Lastly, the research shows that authentic native language data should be offered to Chinese second-language learners. It is suggested that teachers should provide students with some model texts written by native speakers and permit the students to make close observation on how native speakers make the coherent relations evident by using those adverbial connectors. Of course, there are still some limitations in the research which restricts the correctness and preciseness of the research. Hence, much space is still left here to exploit the study further. 


\section{References}

Baker, P., Hardie, A., \& McEnery, T. (2006). A Glossary of Corpus Linguistics. Edinburgh: Edinburgh University Press.

Biber, D., Conrad, S., \& Rappen, R. (2000). Corpus Linguistics: Investigating Language Structure and Use. Beijing: Foreign Language Teaching and Research Press.

Ghadessy, M., Henry, A., \& Roseberry, R. L. (2001). Small Corpus Studies and ELT: Theory and Practice. Amsterdam/ Philadelphia: John Benjamins Publishing Company.

Halliday, M. A. K. (1994). An Introduction to Functional Grammar. London: Edward Arnold Publishers Limited.

Halliday, M. A. K., \& Hasna, R. (1976). Cohesion in English. London: Longman Press.

HE, A. P. (2004a). Corpus Linguistics and English Teaching. Beijing: Foreign Language Teaching and Research Press.

HE, A.P. (2004b). The Application of Corpus in Foreign Language Teaching: Theory and Practice. Guangzhou: Guangdong High Education Press.

Kennedy, G. (2000). An Introduction to Corpus Linguistics. Beijing: Foreign Language Teaching and Research Press.

Quirk, R. (1972). A Grammar of Contemporary English. London: Longman Press.

Sinclair, J. (1991). Corpus, Concordance, Collection. Oxford: Oxford University Press.

Kopple, V. W. J. (1980). Experimental Evidence for Functional Sentence Perspective (Unpublished Doctoral Dissertation, University of Chicago.)

Yang, H. Z. (2004). An Introduction of Corpus Linguistics. Shanghai: Shanghai Foreign Language Education Press. 